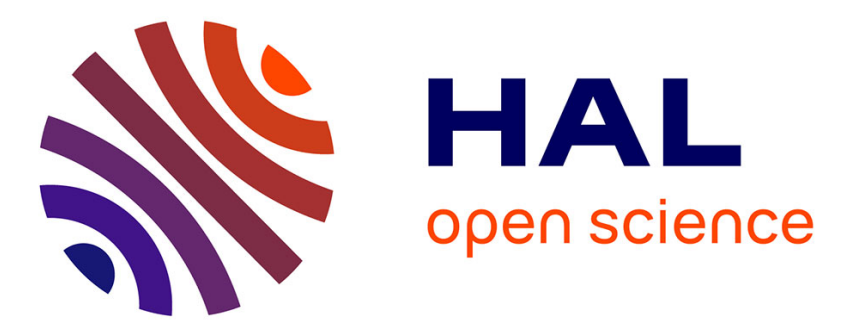

\title{
Risque d'accident des usagers de deux-roues motorisés associé aux pratiques de remontée de files sur autoroutes urbaines
}

Nicolas Clabaux, Jean-Yves Fournier, Jean Emmanuel Michel, Jérôme Huillet, Fabien Gemy, Françoise Gueritat

\section{To cite this version:}

Nicolas Clabaux, Jean-Yves Fournier, Jean Emmanuel Michel, Jérôme Huillet, Fabien Gemy, et al.. Risque d'accident des usagers de deux-roues motorisés associé aux pratiques de remontée de files sur autoroutes urbaines. RTS - Recherche Transports Sécurité, 2016, 2015 (03-04), pp.173-184. 10.4074/S0761898016004039 . hal-01264286v2

\section{HAL Id: hal-01264286 \\ https://hal.science/hal-01264286v2}

Submitted on 12 Feb 2018

HAL is a multi-disciplinary open access archive for the deposit and dissemination of scientific research documents, whether they are published or not. The documents may come from teaching and research institutions in France or abroad, or from public or private research centers.
L'archive ouverte pluridisciplinaire HAL, est destinée au dépôt et à la diffusion de documents scientifiques de niveau recherche, publiés ou non, émanant des établissements d'enseignement et de recherche français ou étrangers, des laboratoires publics ou privés. 


\title{
Risque d'accident des usagers de deux-roues motorisés associé aux pratiques de remontée de files sur autoroutes urbaines
}

\section{Powered two-wheelers drivers' crash risk associated with filtering on urban freeways}

\author{
Nicolas Clabaux · Jean-Yves Fournier · Jean-Emmanuel Michel · Jérôme Huillet · Fabien Gémy · Françoise Guéritat \\ (C) IFSTTAR et Éditions NecPlus 2015
}

\author{
Nicolas Clabaux $(\bowtie)$ \\ Institut Français des Sciences et Technologies des Transports, de \\ l'Aménagement et des Réseaux (Ifsttar) \\ Département Transport Santé Sécurité (TS2) \\ Laboratoire Mécanismes d'Accidents \\ e-mail : nicolas.clabaux@ifsttar.fr \\ Jean-Yves Fournier $(\bowtie)$ \\ Institut Français des Sciences et Technologies des Transports, de \\ l'Aménagement et des Réseaux (Ifsttar) \\ Département Transport Santé Sécurité (TS2) \\ Laboratoire Mécanismes d'Accidents \\ e-mail : jean-yves.fournier@ifsttar.fr

\section{Jean-Emmanuel Michel $(\bowtie)$} \\ Institut Français des Sciences et Technologies des Transports, de \\ l'Aménagement et des Réseaux (Ifsttar) \\ Département Transport Santé Sécurité (TS2) \\ Laboratoire Mécanismes d'Accidents \\ e-mail : jean-emmanuel.michel@ifsttar.fr

\section{Jérôme Huillet $(\square)$} \\ Centre d'études et d'Expertise sur les Risques, l'Environnement, la \\ Mobilité et l'Aménagement (Cerema), \\ direction territoriale Méditerranée \\ Département Conception et Exploitation Durables des Infrastructures \\ (DCEDI) \\ Service Sécurité et Signalisation Routières (SSR) \\ e-mail : jerome.huillet@cerema.fr
}

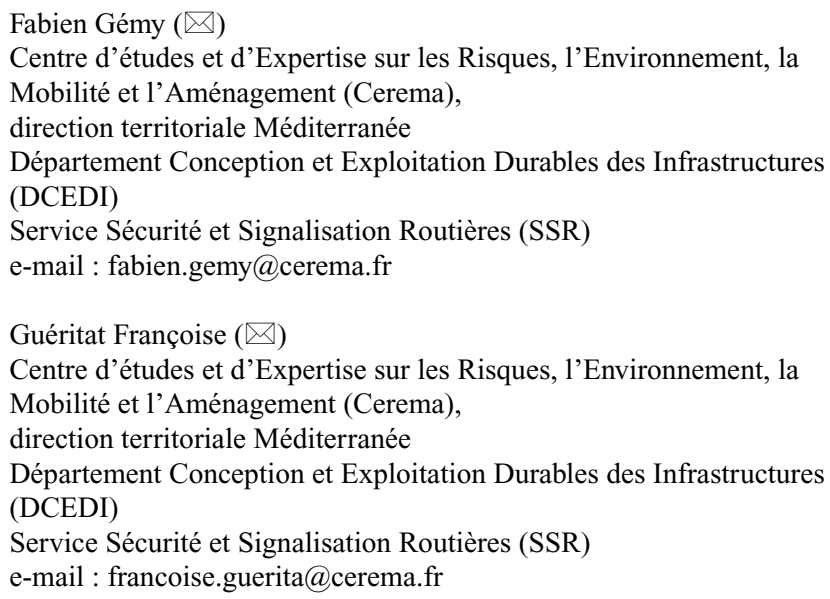

Résumé L'usage croissant des deux-roues motorisés dans les grandes agglomérations pose la question des risques associés à certaines pratiques propres à ce mode de déplacement, comme par exemple la remontée de files de voitures. Cet article propose une contribution à l'estimation du risque d'accident associé aux pratiques de remontée de files sur autoroute urbaine. Les investigations ont porté sur les autoroutes urbaines de l'aire métropolitaine marseillaise. En s'appuyant sur une campagne annuelle d'observations des pratiques de remontée de files des deux-roues motorisés et sur l'analyse des accidents recensés par la police au cours des années 2004 à 2011, ce travail montre que les motocyclistes remontant les files de véhicules ont un risque d'être impliqué dans un accident corporel 2,63 fois (Intervalle de confiance (IC) à $95 \%$ [1,003; 6,89]) supérieur au risque de ceux ne remontant pas les files de véhicules. Ce risque relatif s'élève à 2,71 (I.C. à $95 \%[1,06 ; 6,95])$ pour ceux remontant les files en empruntant l'espace interfiles. L'analyse des cas d'accident suggère qu'une partie de ce sur-risque pourrait provenir du positionnement singulier des deux-roues motorisés sur la chaussée lorsqu'ils remontent les files. Ce positionnement les rendrait moins détectables par les automobilistes que lorsqu'ils circulent dans les files.

Mots clés deux-roues motorisés · motocycliste · remontée de files $\cdot$ interfiles $\cdot$ accident $\cdot$ risque

Abstract The increasing use of powered two-wheelers in large urban areas raises the question of the risks related to certain practices unique to this transportation method, such as filtering. This article proposes a contribution to the estimation of accident risks related to filtering on urban expressways. Our investigations dealt with urban expressways in the Marseille metropolitan area. Taking advantage of an annual campaign of observations of filtering practices by powered two-wheelers and an analysis of accidents recorded by the police during the years 2004 to 2011, this study demonstrates that motorcyclists who do filtering have a risk of being involved in a bodily injury accident that is 
2.63 times $(95 \%$ CI $[1.003 ; 6.89])$ greater than the risk run by those who drive in the lanes. This relative risk reaches $2.71(95 \%$ CI $[1.06 ; 6.95])$ for those who drive in the space between lanes (lane splitting). The analysis of accident cases suggests that this excess risk could be due to the original positioning of powered two-wheelers on the carriageway when they do filtering. This positioning make them less detectable for automobile drivers than when the drive in the lanes.

Keywords Powered two-wheelers · Motorcyclist · Filtering · Lane-splitting $\cdot$ Crash risk $\cdot$ Crash

\section{Introduction}

Face aux problèmes de congestion des réseaux de circulation des grandes agglomérations, l'une des stratégies d'adaptation des individus réside dans le recours au deux-roues motorisé [1]. Le deux-roues motorisé peut en effet permettre dans les grandes agglomérations, de réduire de près de moitié les temps de parcours par rapport à l'automobile [2]. Cela tient en grande partie à la capacité qu'offre ce mode de déplacement à se faufiler dans le trafic et à remonter les files de véhicules arrêtés ou ralentis. Cette pratique de remontée de files de véhicules, peut dans certains espaces très congestionnés, comme en Île-de-France, représenter la majeure partie des trajets. Aupetit et al. [3] montrent, par exemple, qu'elle représente en moyenne, parmi 16 motocyclistes suivis au cours de leurs trajets domicile-travail, $72 \%$ du temps de parcours et $77 \%$ de la distance parcourue. Cette pratique est cependant perçue comme à risque par les usagers de deux-roues motorisés, comme par les automobilistes [3, 4]. Les études d'accidents disponibles dans la littérature scientifique internationale montrent qu'en effet il s'agit d'une pratique à risque et qui donne lieu à la production d'accidents (voir notamment : [3, 5, 6, 7, 8]). Clarke et al. [6] montrent, par exemple, qu'un accident de deux-roues motorisé sur vingt recensés en Grande-Bretagne implique un deux-roues motorisé en train de remonter une file de voitures. En France, et pour ce qui concerne uniquement les remontées de files par les espaces entre les files de circulation, Aupetit et al. [3] estiment en s'appuyant sur le fichier national des accidents corporels, que, $3,4 \%$ des motocyclistes impliqués dans un accident, circulaient entre deux files au moment de l'accident. Pour la seule région Ile-de-France, l'enjeu s'élève à $7,3 \%$ des accidents (même référence) ${ }^{1}$. Mais, au-delà de l'enjeu en nombre d'accidents que les remontée de files représentent, la question qui est parfois posée est de savoir

\footnotetext{
${ }^{1}$ Ces enjeux sont probablement sous-estimés puisque la modalité « entre deux files » de la variable « manœuvre principale avant l'accident » semble peu renseignée dans les fiches BAAC (Bulletin d'analyse des accidents corporels) par les forces de l'ordre ( $c f$. point 3.3 de cet article et [10])
}

si le risque d'accident associé à cette pratique est supérieur, équivalent ou inférieur au risque encouru lorsque les usagers de deux-roues ne remontent pas les files de véhicules et restent dans le flux de trafic, dans les voies de circulation (voir par exemple : [9]). Hormis les investigations réalisées par Ouellet [9], il n'existe pas, à notre connaissance, de travaux récents permettant de répondre à cette question. L'objectif de cet article est de contribuer à combler cette lacune. Nous rendons compte ci-après d'une recherche réalisée sur l'aire métropolitaine marseillaise et qui vise à mieux connaître le risque d'accident des usagers de deux-roues motorisés en situation de remontée de files. Nous nous focalisons dans ce papier sur les pratiques de remontée de files sur les autoroutes urbaines. Nous traiterons dans un autre article du risque associé à la pratique de remontée de files sur voies urbaines.

\section{Démarche et aspects de méthode}

Dans cet article, nous cherchons à estimer le risque d'accident des usagers de deux-roues motorisés remontant les files de véhicules sur les autoroutes urbaines de l'aire métropolitaine marseillaise au cours des heures de pointe du matin et du soir. La démarche adoptée a donc consisté à chercher à mettre en relation :

- d'une part un nombre d'usagers de deux-roues motorisés impliqués dans un accident corporel alors qu'ils étaient en train de remonter une file de véhicules, sur une autoroute urbaine ;

- d'autre part une exposition au risque, c'est-à-dire le nombre de kilomètres parcourus par les usagers de deux-roues motorisés en remontée de files au cours des mêmes périodes et sur les mêmes axes ${ }^{2}$.

Nous comparons ensuite ce risque à celui des conducteurs de deux-roues motorisés ne remontant pas les files de véhicules sur les mêmes axes et aux mêmes heures.

Les investigations ont porté sur les autoroutes urbaines de l'aire métropolitaine marseillaise. Avec plus de 1,5 million d'habitants, l'aire métropolitaine marseillaise est l'une des douze aires métropolitaines que compte le territoire français. Située sur la côte méditerranéenne, l'usage des deux-roues motorisés y est depuis longtemps assez important. L'accidentalité y est également particulièrement

\footnotetext{
${ }^{2}$ Nous avons en effet choisi de mesurer l'exposition au risque par les kilomètres parcourus par les motocyclistes en remontée de files. Cette mesure de l'exposition au risque n'est certes pas sans inconvénient [12]. Elle ne tient par exemple par compte de la vitesse de circulation qui est probablement différente entre les motocyclistes remontant les files et ceux restant dans le flot de circulation. Elle était cependant plus facile à collecter par rapport par exemple au temps passé par les motocyclistes en remontée de files. Elle est d'autre part très répandue dans la littérature scientifique internationale en sécurité routière [13].
} 
élevée. La ville de Marseille est en effet, après Paris, la ville qui concentre le plus d'accidents de deux-roues motorisés. En outre, le département des Bouches-du-Rhône est, en effectifs d'accidents de deux-roues motorisés, le deuxième département le plus concerné, après Paris ${ }^{3}$.

Les investigations ont porté sur l'autoroute A7 reliant Vitrolles à Marseille, l'autoroute A50 reliant Aubagne et Toulon à Marseille, l'autoroute A51 reliant Aix-en-Provence à Marseille via l'A7 et l'autoroute A55 reliant l'ouest de l'étang de Berre à Marseille (voir Fig. 1). Ces axes constituent les pénétrantes principales de l'agglomération de Marseille et supportent d'importants niveaux de trafic (voir Fig. 1). Ils comportent généralement trois voies pour chaque sens et la vitesse réglementaire y est en général limitée à $90 \mathrm{~km} / \mathrm{h}$. Ces sections se caractérisent également par une forte densité de bretelles de sortie et de voies d'entrée, ce qui est une source de perturbation de l'écoulement du trafic, en particulier aux heures de pointe.

L'objectif étant d'estimer sur ces axes le risque des usagers de deux-roues motorisés en situation de remontée de files, un préalable était d'identifier pour ces autoroutes urbaines, les périodes au cours desquelles le trafic est régulièrement congestionné et les sections sur lesquelles interviennent ces périodes de congestion. Nous avons considéré qu'une section est congestionnée lorsque le trafic s'écoule sur l'ensemble des voies à une vitesse moyenne inférieure ou égale à $60 \mathrm{~km} / \mathrm{h}$. Cette limite est celle généralement utilisée dans la littérature technique pour considérer une infrastructure autoroutière congestionnée [11]. Il fallait que cette congestion s'étende sur une section d'au moins 500 mètres et dure au moins une heure pour que la section soit incluse dans l'étude. Pour identifier ces sections et périodes où le trafic est congestionné, nous nous sommes appuyés sur les données collectées sur les conditions de trafic par les stations Siredo de la Dirmed (Direction Interdépartementale des Routes Méditerranée) implantées à intervalle régulier sur le réseau autoroutier desservant la ville de Marseille (tous les 500 mètres environ). L'étude détaillée des débits et des vitesses moyennes par séquence de six minutes au cours des jours de semaine de quatre semaines de l'année 2012 (une semaine de chaque trimestre), nous a amené à identifier 12 sections congestionnées réparties sur quatre autoroutes distinctes (voir Fig. 1). Le tableau 1 présente les 12 sections congestionnées étudiées. Chaque section congestionnée est définie par trois caractéristiques principales :

- sa localisation (et sa longueur), sur un tronçon d'autoroute délimité par un Point de Repère (PR) de début et un PR de fin,

- un sens de circulation (sens « entrant» vers Marseille et sens « sortant»),

${ }^{3}$ Source : Fichier national des accidents corporels (année 2013).
- une tranche horaire (matin ou soir) durant laquelle le trafic est congestionné et durant laquelle les observations ont été réalisées.

Pour chacune de ces sections congestionnées étudiées, nous avons cherché à connaître, d'une part l'exposition au risque des usagers de deux-roues motorisés, d'autre part le nombre d'accidents corporels recensés. Afin de disposer d'effectifs d'accidents suffisants, nous avons fait porter les investigations sur les années 2004 à 2011. Le choix de ces années a été conditionné par la disponibilité de données à la fois sur les trafics et les accidents. Nous présentons ci-après la démarche que nous avons adoptée pour la collecte de ces deux catégories de données.

\subsection{Collecte des données d'exposition au risque}

Afin de connaître le nombre de deux-roues motorisés $\mathrm{x}$ kilomètres exposés au risque d'accident en situation de remontée de files et en situation de non remontée de file sur les sections et périodes retenues, nous avons dans un premier temps cherché à connaître pour chaque année étudiée (de 2004 à 2011), le trafic total qui s'est écoulé sur chacune des sections et au cours de chaque tranche horaire étudiée. Nous nous sommes pour cela appuyés sur les données de trafic collectées par les stations Siredo et sur les évolutions de ces trafics d'une année sur l'autre.

Nous avons ensuite cherché à connaître la part que représentaient les deux-roues motorisés dans ces trafics. Une campagne d'observations du trafic deux-roues motorisés a pour cela été conduite tout au long de l'année 2013. Le choix des dates et des points d'observations s'est fait selon les étapes suivantes :

Pour chaque section congestionnée :

i. identification des différents points d'observations possibles. Chaque point devait surplomber la section étudiée et offrir de bonnes conditions de visibilité, en amont et en aval du point d'observation. Il s'agissait généralement de ponts, plus rarement de bâtiments, de talus ;

ii. tirage aléatoire parmi l'ensemble des jours de semaine (du lundi au vendredi) de l'année 2013, hors jours fériés, de trois dates d'observations ;

iii. pour chaque date tirée, tirage aléatoire, d'un point d'observation parmi l'ensemble des points d'observation possibles.

36 séquences d'observations réparties sur toute l'année 2013 sur les différentes sections et tranches horaires étudiées, ont donc été réalisées. Ces observations ont été conduites depuis 19 points d'observations distincts (voir Fig. 1) par des équipes de deux enquêteurs, qui recensaient le nombre de deux-roues motorisés passant devant eux. Pour 


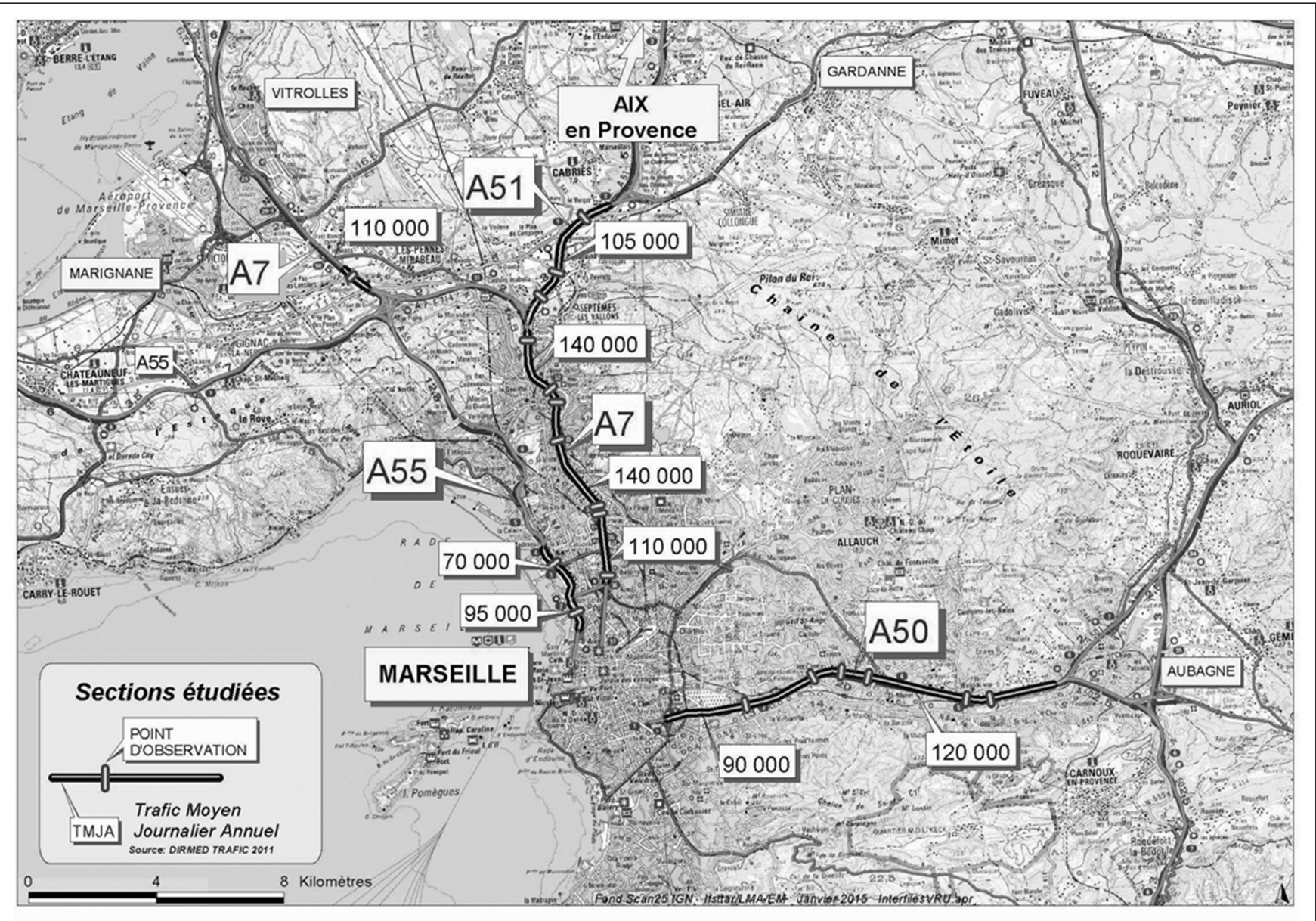

Fig. 1 Aire métropolitaine marseillaise, tronçons d'autoroutes urbaines étudiés et ordre de grandeur des Trafics moyens journaliers annuels (TMJA dans les deux sens)

Tableau 1 Principales caractéristiques des sections congestionnées étudiées

\begin{tabular}{|c|c|c|c|c|c|c|}
\hline $\begin{array}{l}\text { Sections } \\
\text { congestionnées }\end{array}$ & Autoroute & Sens/Marseille & Périodes étudiées & $\begin{array}{l}\mathrm{PR} \text { * début } \\
\text { de section }\end{array}$ & $\begin{array}{l}\text { PR* } \\
\text { fin de section }\end{array}$ & $\begin{array}{l}\text { Longueur de la } \\
\text { section (en km) }\end{array}$ \\
\hline Section 1 & A7 & Sortant & 07 h $15-09$ h 30 & 266.000 & 264.000 & 2,0 \\
\hline Section 2 & A7 & Entrant & 07 h $00-09$ h 30 & 271.500 & 274.500 & 3,0 \\
\hline Section 3 & A7 & Entrant & 07 h $15-08$ h 45 & 274.500 & 280.000 & 5,5 \\
\hline Section 4 & A7 & Entrant & 17 h $00-19$ h 00 & 271.500 & 280.000 & 8,5 \\
\hline Section 5 & A7 & Sortant & 07 h $00-08$ h 45 & 280.000 & 271.500 & 8,5 \\
\hline Section 6 & A7 & Sortant & 17 h $00-18$ h 30 & 280.000 & 271.500 & 8,5 \\
\hline Section 7 & A50 & Entrant & 07 h $00-09$ h 00 & 013.000 & 0.000 & 13,0 \\
\hline Section 8 & A50 & Entrant & 17 h $00-18$ h 30 & 013.000 & 0.000 & 13,0 \\
\hline Section 9 & A51 & Entrant & 07 h $15-09$ h 15 & 006.000 & 0.000 & 6,0 \\
\hline Section 10 & A51 & Entrant & 17 h $30-19$ h 00 & 006.000 & 0.000 & 6,0 \\
\hline Section 11 & A55 & Entrant & 07 h $00-09$ h 30 & 004.000 & 0.800 & 3,2 \\
\hline Section 12 & A55 & Entrant & 16 h $30-20$ h 00 & 004.000 & 0.800 & 3,2 \\
\hline
\end{tabular}

Necplus 
chaque deux-roues motorisé, ils notaient également s'il effectuait ou non une remontée de files et l'espace utilisé pour effectuer cette remontée de files (interfiles de gauche, interfiles de droite, bande d'arrêt d'urgence ou tout autre espace disponible comme, par exemple, la bande dérasée de gauche). Un deux-roues motorisé était considéré comme remontant une file de véhicules dès lors qu'il dépassait, de façon consécutive, au moins deux véhicules dans une zone d'environ 100 mètres précédant ou suivant, selon les cas, le point d'observation.

Rappelons ici qu'en France, la remontée de files est une pratique interdite par le code de la route. Elle est cependant largement répandue dans les grandes agglomérations. Elle fait d'autre part l'objet de peu de contrôle et de répression par les forces de l'ordre [14].

Prises dans leur ensemble, ces observations nous ont permis de connaître la proportion de deux-roues motorisés dans le trafic total en 2013 sur l'ensemble des sections congestionnées étudiées. Nous avons considéré que cette proportion était la même de 2004 à 2011. Nous avons donc fait l'hypothèse que cette proportion n'a pas évolué entre l'année 2013 et les années 2004 à 2011. En effet, nous ne disposions pas de données sur les trafics deux-roues motorisés au cours de la période 2004-2011. Cette hypothèse nous a néanmoins paru acceptable ${ }^{4}$.

Concernant la proportion de deux-roues motorisé en remontée de files et la proportion de deux-roues motorisés circulant dans les voies de circulation sans remonter les files, nous avons considéré pour chaque section, que la proportion moyenne observée au cours des trois séquences d'observations était représentative des pratiques de remontée de file et de non-remontée de file sur cette section pour les années 2004 à 2011. En d'autres termes, nous avons considéré que la fréquence de cette pratique n'a pas évolué entre l'année 2013 où les observations ont été conduites et la période 2004-2011. Il s'agit d'une hypothèse peut-être légèrement abusive. Fin novembre 2013, le conseil national de sécurité routière, a en effet proposé

\footnotetext{
${ }^{4}$ Nous avons considéré cette hypothèse comme acceptable dans la mesure où l'augmentation de la pratique du deux-roues motorisé constatée en France et dans les Bouches-du-Rhône au cours des années 2000 , concerne surtout les motocyclettes légères et dans une moindre mesure les motocyclettes lourdes. Or le réseau que nous étudions est surtout fréquenté par des motocyclettes lourdes (voir plus loin, tableau 2). Par exemple, dans les Bouches-du-Rhône, 4249 motos lourdes ont été immatriculées en 2013, contre 4115 en 2004 (soit une hausse de $3,3 \%$ ). Il y a bien eu une augmentation substantielle des ventes de 2006 à 2008 pour atteindre 5068 motos lourdes immatriculées en 2008, mais cette augmentation a été suivie d'une baisse du même ordre de grandeur jusqu'en 2013 (source : Officiel du cycle, de la moto et du quad). Par ailleurs, le nombre de permis de conduire une motocyclette lourde délivré en France, reste relativement stable sur la période 2004-2012 passant de 102785 permis délivrés en 2004 à 116889 en 2012 (source : SOES).
}

une expérimentation d'une autorisation de cette pratique sur les voies autoroutières urbaines de quatre grandes agglomérations. Cette expérimentation, qui devrait débuter en 2016, fait suite à un rapport d'un groupe de travail remis au ministre de l'intérieur en novembre 2012, qui concluait dans le sens d'une légalisation et d'un encadrement de cette pratique. La presse spécialisée a évoqué au cours de l'année 2013 ce rapport et cette expérimentation à venir. Il est possible que cela ait eu pour effet d'accroître la pratique de la remontée de files sur le réseau étudié et que par conséquent, les pratiques observées en 2013 aient été plus fréquentes qu'au cours des années 2004 à 2011. Si tel était le cas, les risques d'accident associé à cette pratique que nous obtiendrons devront donc être considérés comme des minimums.

Enfin, notons que sur certaines des sections étudiées (7 sur 12), la limitation de vitesse a été abaissée en 2012, passant de $110 \mathrm{~km} / \mathrm{h}$ à $90 \mathrm{~km} / \mathrm{h}^{5}$. Cette limitation a probablement eu un impact sur les conditions de trafic (baisse des vitesses pratiquées, modification de la congestion, notamment). Cet impact a cependant sans doute été moindre au cours des heures de pointe du soir et du matin, périodes sur lesquelles porte cette recherche.

Pour chaque section congestionnée étudiée, nous avons ensuite appliqué les proportions obtenues aux trafics totaux issus des stations Siredo. Cela nous a permis d'estimer pour chaque année, pour chaque section étudiée et au cours des tranches horaires étudiées, le nombre de deux-roues motorisés qui ont circulé en remontant les files de véhicules et le nombre de deux-roues motorisés qui ont circulé en restant dans le flux de trafic.

Afin d'exprimer l'exposition au risque en conducteurs $\mathrm{x}$ kilomètres et afin de tenir compte de la longueur des sections dans le calcul du risque, nous avons multiplié pour chaque section, les effectifs de deux-roues motorisés par la longueur, en kilomètres, de la section.

Une fois l'exposition au risque connue, nous avons cherché à connaître le nombre de deux-roues motorisés qui ont été accidentés entre 2004 et 2011 sur les sections étudiées et au cours des tranches horaires retenues, soit alors qu'ils remontaient une file de véhicules, soit alors qu'ils circulaient dans les voies de circulation. Nous présentons ci-après la démarche adoptée pour déterminer ces effectifs.

\subsection{Collecte des données d'accidents}

Nous nous sommes appuyés sur les accidents corporels recensés par la Compagnie républicaine de sécurité

\footnotetext{
${ }^{5}$ Direction Interdépartementale des Routes Méditerranée. Juin 2012. Dossier de presse. Réduction des vitesses sur les autoroutes non concédées des Bouches-du-Rhône. http://www.enroute.mediterranee.equipement.gouv.fr (accès le 12 février 2015).
} 
autoroutière Provence (CRS-AP) intervenant sur les sections étudiées. Entre 2004 et 2011, 101 accidents corporels impliquant au moins une motocyclette ont été recensés sur les sections et tranches horaires étudiées. Parmi ces 101 cas d'accident, 9 cas n'ont pas été retrouvés dans les archives de la Compagnie républicaine de sécurité autoroutière Provence du secteur d'étude. Parmi les 92 cas d'accidents restants qui impliquent 97 motocyclettes, 42 cas ont donné lieu à un procès-verbal détaillé et 50 cas ont donné lieu à un procès-verbal simplifié. Chaque procès-verbal a fait l'objet d'un examen approfondi par trois experts en accidentologie du Laboratoire Mécanismes d'Accidents de l'Ifsttar. Ce travail a été réalisé de façon indépendante par les trois experts afin de pouvoir examiner la robustesse de leurs analyses. Ces experts devaient notamment se prononcer pour chaque cas, sur la manœuvre réalisée par le deux-roues motorisé au moment de l'accident et en particulier s'il était en situation de remontée de files ou non. Si l'accident impliquait deux deux-roues motorisés ( 5 cas sur 92), les experts devaient se prononcer sur la manœuvre de chacun des deux-roues motorisés. Chacun des 97 deux-roues motorisés impliqués était affecté à l'une des catégories suivante :

0. si l'expert était dans l'incapacité de se prononcer sur la manœuvre du deux-roues au moment de l'accident $\mathrm{du}$ fait du manque d'informations disponibles dans la procédure $^{6}$;

1. si l'expert jugeait que le deux-roues circulait dans les voies de circulation, sans remonter une file de véhicules ;

2. si l'expert jugeait que le deux-roues était probablement en train de circuler dans les voies sans remonter une file de véhicules ;

3. si l'expert jugeait que le deux-roues motorisé était probablement en train de remonter une file de véhicules au moment de l'accident ;

4. si l'expert jugeait que le deux-roues motorisé était en train de remonter une file de véhicules au moment de l'accident.

Notons que pour les cas affectés aux catégories 3 ou 4, les experts notaient également l'espace emprunté par le deux-roues motorisé pour remonter la file de véhicules.

Une fois ces investigations réalisées, un taux d'agrément inter-experts a été calculé. Les différentes catégories possibles étant mutuellement exclusives, nous avons eu recours au coefficient d'agrément Kappa de Fleiss [15]. Puis, les éventuels cas de désaccord ont donné lieu à une harmonisation entre les experts afin que chaque cas soit attribué

${ }^{6}$ Ces cas ont ensuite été écartés des analyses. à une catégorie $(0,1,2,3$ ou 4). En l'absence de consensus entre les experts, le cas était considéré comme indéterminé.

$\mathrm{Au}$ final, l'ensemble de ces investigations nous ont permis de connaître pour chaque année et pour chaque section congestionnée, d'une part le nombre de deux-roues motorisé $\mathrm{x}$ kilomètres en remontée de files et le nombre de deux-roues $\mathrm{x}$ kilomètres hors remontée de files; et d'autre part le nombre de deux-roues motorisés accidentés sur les mêmes sections et au cours des mêmes périodes alors qu'ils étaient en train d'effectuer ou non une remontée de files. Il nous était alors possible d'estimer le risque d'accident par kilomètre parcouru des usagers remontant les files de véhicules et le risque d'accident par kilomètre parcouru des usagers de deux-roues motorisés ne remontant pas les files de véhicules.

\subsection{Méthode pour l'estimation des risques d'accident}

Pour chacune des sections congestionnées et pour la période 2004-2011, nous avons dans un premier temps estimé :

- le risque d'accident des conducteurs de deux-roues remontant les files de véhicules (rapport entre le nombre de conducteurs de deux-roues à moteur impliqués dans un accident corporel alors qu'ils remontaient une file de véhicules et le nombre de conducteurs de deux-roues à moteur $\mathrm{x}$ kilomètres exposés à ce risque) et son intervalle de confiance à $95 \%$;

- le risque d'accident des conducteurs ne remontant pas les files de véhicules et son intervalle de confiance à $95 \%$.

Dans un second temps, nous avons estimé le Risque relatif (RR) de ces deux populations en utilisant la formule suivante :

$$
\mathrm{RR}=\frac{\begin{array}{c}
\mathrm{Nb} \text { de DRMaccidentés en remontée de files/ } \\
\mathrm{Nb} \text { de DRM } \times \text { km en remontée de files }
\end{array}}{\begin{array}{c}
\mathrm{Nb} \text { de DRM accidenté s hors remontée de files/ } \\
\mathrm{Nb} \text { de DRM } \times \text { km horsremontée de files }
\end{array}}
$$

Enfin, les effectifs d'accidents n'étant pas nécessairement suffisants sur chaque section pour obtenir un résultat significatif, nous avons estimé un risque relatif commun à l'ensemble des sections et son intervalle de confiance à $95 \%$ en procédant à une méta-analyse (voir : [16] p. 15 et suivantes). Chaque section est alors analysée de façon indépendante. Nous avons évalué l'hétérogénéité statistique des résultats entre les sections par la statistique $\mathrm{Q}$ de DerSimonian et Laird [17, 18].

Nous présentons ci-après les résultats de ces investigations. Nous commençons par quelques résultats issus des campagnes d'observation et des analyses d'accidents. 


\begin{tabular}{|c|c|c|}
\hline & $\begin{array}{l}\text { Nombre de } \\
\text { véhicules } \\
\text { observés }\end{array}$ & $\begin{array}{l}\text { \% du trafic } \\
\text { deux-roues } \\
\text { motorisés }\end{array}$ \\
\hline Motocyclettes légères & 2273 & $24,2 \%$ \\
\hline dont type scooter & 1852 & $19,7 \%$ \\
\hline dont type moto & 421 & $4,5 \%$ \\
\hline Motocyclettes lourdes & 6537 & $69,6 \%$ \\
\hline dont type scooter & 1262 & $13,4 \%$ \\
\hline dont type moto & 5275 & $56,2 \%$ \\
\hline Trois-roues motorisés & 582 & $6,2 \%$ \\
\hline $\begin{array}{l}\text { Total deux/trois-roues } \\
\text { motorisés }\end{array}$ & 9392 & \\
\hline
\end{tabular}

\section{Résultats}

\subsection{Résultats concernant les trafics}

Au cours des 36 séquences d'observation réalisées, 9392 deux-roues motorisés ont été observés par les enquêteurs. Rapportés au nombre total de véhicules circulant, les deuxroues motorisés représentent en moyenne sur les sections retenues et en heure de pointe, 3,4 \% du trafic. Parmi les deux-roues motorisés observés, les Motocyclettes lourdes (MTT) sont majoritaires (voir tableau 2). Elles représentent $69,6 \%$ des deux-roues motorisés. Les Motocyclettes légères $(\mathrm{MTL})^{7}$ représentent $24,2 \%$ de ce trafic. Les tricycles à moteur de type «pendulaire » (e.g. Piaggio MP3) représentent $6,2 \%$. Dans la suite du document, les tricycles, qui, dans la grande majorité des cas ont une cylindrée supérieure à $125 \mathrm{~cm}^{3}$, seront intégrés aux motocyclettes lourdes.

Concernant les conditions de circulation pendant les séquences d'observation, deux principaux groupes de sections se distinguent.

\footnotetext{
${ }^{7}$ En France, les motocyclettes sont séparées en deux catégories administratives principales : (i) les motocyclettes légères, de cylindrée supérieure à $50 \mathrm{~cm}^{3}$ et inférieure à $125 \mathrm{~cm}^{3}$. Elles peuvent être conduites avec un permis voiture sous certaines conditions Il s'agit très généralement de scooters. Les autres catégories (basique, custom, trail, sportive) sont minoritaires ; (ii) les motocyclettes lourdes, de cylindrée supérieure à $125 \mathrm{~cm}^{3}$ qui nécessitent un permis moto. On distingue sept principales catégories de motocyclettes lourdes : custom, basique, roadster, grand-tourisme, sportive, trail, scooter. Les scooters sont minoritaires.
}

D'une part, les sections où le trafic est très ralenti pendant presque toute la durée de la période d'observation. Il s'agit des sections 9, 11 et 12 (voir tableau 1). La vitesse du trafic sur l'ensemble des voies est en moyenne comprise entre 20 et $50 \mathrm{~km} / \mathrm{h}$.

D'autre part, les sections où le flux de véhicules est irrégulier. Se succèdent des séquences où le trafic est arrêté ou au ralenti pendant quelques minutes et des séquences où le trafic est dense, avec peu d'espace entre les véhicules, mais s'écoule à une vitesse relativement importante, qui peut parfois même approcher la limite réglementaire de $90 \mathrm{~km} / \mathrm{h}$. Sur ces sections, contrairement à ce qu'on avait constaté sur les données de trafic des quatre semaines de l'année 2012 ( $c f$. point 2), la vitesse moyenne du trafic pendant les séquences d'observation est généralement supérieure à $60 \mathrm{~km} / \mathrm{h}$, comprise entre $57 \mathrm{~km} / \mathrm{h}$ et $89 \mathrm{~km} / \mathrm{h}$. Ces sections sont les sections : 1, 2, 3, 4, 5, 6, 7, 8 et 10 (voir tableau 1).

Par conséquent, les résultats que nous présentons ci-après sur les pratiques de remontée de files et les risques associés à ces pratiques, correspondent à des conditions de trafic hétérogènes que l'on retrouve aux heures de pointe sur les autoroutes urbaines desservant Marseille, allant de situations où le trafic est à l'arrêt ou au ralenti à des situations, par ailleurs plus fréquentes, où le trafic est chargé, dense, mais s'écoule à une vitesse relativement importante. On retrouve cette hétérogénéité des conditions de trafic dans les études antérieures traitant des pratiques de remontée de files sur voies autoroutières et portant sur d'autres terrains (voir notamment : $[3,9,19]$ ).

\subsection{Résultats sur les pratiques de remontée de files des usagers de deux-roues motorisés}

Parmi les 9392 usagers de deux-roues motorisés, près d'un usager sur trois $(32,1 \%)$ était en train de remonter une file de véhicules lorsqu'il a été observé. Parmi les usagers de motocyclettes lourdes, cette proportion s'élève à 39,3\%. Parmi les usagers de motocyclettes légères, elle s'élève à 26,8\%. La différence est statistiquement significative $\left(\mathrm{X}^{2}=21,7 \mathrm{p}<0,01\right)$.

D'un point de vue qualitatif, la vitesse du flux de trafic influence fortement la pratique de la remontée de files. Lorsque la vitesse du flux est relativement importante (supérieure à $70 \mathrm{~km} / \mathrm{h}$ ), moins d'un deux-roues motorisé sur cinq remonte les files. Il s'agit alors pour l'essentiel d'usagers de motocyclettes lourdes. Lorsqu'elle est plus faible (inférieure à $50 \mathrm{~km} / \mathrm{h}$ ), près de quatre deux-roues motorisés sur cinq remontent les files de véhicules. Concernant l'effet de la vitesse du flux de trafic sur les pratiques de remontée de files, nous renvoyons le lecteur aux références [9] ou [19], où des investigations plus poussées ont été réalisées et confirment ce lien. 
On constate des écarts dans la proportion des manœuvres de remontées de files de deux-roues motorisés selon les sections. Ces écarts traduisent surtout des différences dans le niveau de congestion entre les sections. C'est par exemple sur les sections 9,11 et 12 , qui sont les sections les plus congestionnées, que les manœuvres de remontée de files des deux-roues motorisés sont les plus fréquentes. Plus de $75 \%$ des deux-roues motorisés étaient en train de remonter une file de véhicules lorsqu'ils ont été observés.

Concernant l'espace de la chaussée utilisé pour remonter les files de véhicules, l'espace interfiles est majoritaire puisque $71 \%$ des deux-roues motorisés observés en train de remonter une file de voitures, empruntaient l'espace interfiles. Il s'agit quasi-systématiquement de l'espace interfiles situé entre la file de gauche et la voie médiane (interfiles de gauche). Nous ne distinguerons donc pas dans la suite des documents l'espace interfiles de droite et l'espace interfiles de gauche. Viennent ensuite la bande d'arrêt d'urgence qui représente $26 \%$ des situations et les autres espaces disponibles (zébra, bande dérasée) qui représentent $2,5 \%$.

\subsection{Résultats des analyses d'accidents}

L'examen approfondi des 92 cas d'accidents corporels par les trois experts aboutit aux conclusions suivantes :

Le Kappa de Fleiss, mesurant le niveau d'agrément entre les trois experts, s'élève à 0,68 , ce qui peut être considéré comme un bon résultat [15].

Après harmonisation entre les experts, les cas se répartissent de la façon suivante : parmi les 97 motocyclistes impliqués, 36 relèvent de la catégorie 0 . Ces cas sont par la suite écartés des analyses. Parmi les 61 cas restants ; 19 relèvent de la catégorie $1 ; 6$ relèvent de la catégorie $2 ; 8$ relèvent de la catégorie 3 et 28 relèvent de la catégorie 4 .

D'après ces résultats, 28 motocyclistes ont été considérés par les trois experts comme étant en train de remonter une file de véhicules au moment de l'accident sur un total de 61 (soit 46\%) pour lesquels il leurs a été possible de se prononcer sur la manœuvre du deux-roues motorisés. Si on ajoute les cas pour lesquels les trois experts ont estimé la manœuvre de remontée de files du deux-roues comme probable (catégorie 3), la proportion s'élève à 36 sur 61 , soit $59 \%$. Si on compare cette proportion à la proportion des pratiques de remontée de files dans la circulation (voir paragraphe 3.2), on observe que les conducteurs remontant les files sont significativement surreprésentés dans les accidents $\left(X^{2}=18.8 ; p<0.01\right)$.

Concernant les conducteurs de motocyclettes lourdes, parmi les 42 conducteurs impliqués pour lesquels il nous a été possible de se prononcer sur la manœuvre au moment de l'accident, 23 (soit $55 \%$ ) ont été considérés comme étant en remontée de files.
Concernant les conducteurs de motocyclettes légères, parmi les 19 conducteurs impliqués, 13 (soit $68 \%$ ) ont été considérés comme étant en train de remonter une file de véhicules. Là encore, si on compare ces proportions avec la proportion de pratiques de remontées de files des conducteurs de motocyclettes lourdes et de motocyclettes légères qui s'élève respectivement à $39,3 \%$ et $26,8 \%$, cela suggère un sur-risque d'accident à la fois pour les conducteurs de motocyclettes légères $\left(X^{2}=15,5 ; p\right.$ $<0,01)$ et pour les conducteurs de motocyclettes lourdes $\left(X^{2}=4,09 ; p<0,05\right)$ remontant les files de voitures.

Concernant l'espace utilisé par les deux-roues motorisés en remontée de file au moment de l'accident $(n=36), 23$ utilisaient l'espace interfiles ${ }^{8}, 13$ utilisaient la bande d'arrêt d'urgence ou les autres espaces disponibles (zébra, bande dérasée de gauche, etc.).

$\mathrm{Du}$ point de vue des processus à l'œuvre dans les accidents, l'examen approfondi des 56 cas d'accident (impliquant 61 motocyclistes), selon un modèle d'analyse séquentiel de l'accident [20] et le rapprochement des cas jugés similaires sur la base de similitudes d'ensemble dans leur déroulement, montre que cinq principales catégories d'accidents se distinguent. Trois (catégories A, B et $\mathrm{C}$ dans le tableau 3) parmi les accidents impliquant des motocyclistes en remontée de files, deux parmi les accidents impliquant des motocyclistes circulant dans les files (catégories $\mathrm{D}$ et $\mathrm{E}$ ). Le tableau 3 présente ces différentes catégories et les effectifs pour chacune d'entre elles.

\subsection{Résultats des analyses de risque}

Nous présentons dans le tableau 4, pour chaque section et pour l'ensemble de la période étudiée : les effectifs de conducteurs accidentés selon qu'ils étaient ou non en train de remonter une file de véhicules, les estimations du nombre de kilomètres parcourus par les conducteurs de deux-roues motorisés en remontée de files et hors remontée de files ; les risques relatifs d'accidents des deux-roues motorisés en remontée de files par rapport aux deux-roues motorisés hors remontée de files, accompagnés de leur intervalle de confiance à $95 \%$.

Le risque relatif est supérieur à 1 sur 5 des 12 sections étudiées. La borne basse de l'intervalle de confiance est supérieure à 1 sur deux sections. Le risque relatif est inférieur à 1 sur deux sections. Il s'agit de deux des trois sections les plus congestionnées.

Il est donc difficile de tirer des conclusions par section.

\footnotetext{
${ }^{8}$ Notons que parmi les 23 motocyclistes considérés en remontée de files par l'espace interfiles, 8 motocyclistes étaient considérés dans le fichier BAAC comme «entre deux files » (modalité 3 de la variable « manœuvre principale avant l'accident $»)$.
} 
Tableau 3 Principales catégories d'accidents impliquant au moins un motocycliste sur les sections étudiées $(n=56)$

A. Collision entre un véhicule changeant de file (de la gauche vers la droite dans 10 cas sur 15) et un deux-roues motorisé non détecté remontant les files en utilisant l'espace interfiles

B. Collision entre un véhicule rejoignant la bande d'arrêt d'urgence (pour remonter les files ou suite à un problème mécanique) et un deux-roues motorisé non détecté remontant les files par la bande d'arrêt d'urgence.

C. Perte de contrôle d'une motocyclette lourde lors d'une manœuvre rapide de remontée de files par l'espace interfiles. Cette perte de contrôle est souvent liée à un fort ralentissement en aval ou à un véhicule encombrant empiétant sur l'espace interfiles (poids-lourd)

D. Collision arrière entre un deux-roues motorisé circulant dans les files et un ou plusieurs autres véhicules, suite à un ralentissement soudain de la circulation

E. Collision entre un véhicule changeant de file (de la gauche vers la droite dans 6 cas sur 7) et un deux-roues motorisé non détecté circulant dans la file de destination

F. Autres cas

En revanche, la méta-analyse des données des douze sections montre que les conducteurs de deux-roues motorisés remontant les files de véhicules ont un risque d'accident 2,63 fois supérieur (IC à $95 \%[1,003 ; 6,89]$ ) au risque de ceux ne remontant pas les files. La statistique $\mathrm{Q}$ ne conduit pas à rejeter l'hypothèse d'homogénéité entre les sites.

Ce risque s'élève à 2,71 (IC à $95 \%[1,06 ; 6,95]$ ) pour les usagers de deux-roues motorisés empruntant l'espace interfiles. Il n'y a pas non plus dans ce cas d'hétérogénéité significative. Les résultats ne sont pas significatifs concernant les autres espaces utilisées pour pratiquer la remontée de files car les effectifs d'accidents sont trop faibles ${ }^{9}$. En ce qui concerne le risque des différentes catégories de deux-roues motorisés, le risque relatif des conducteurs de motocyclettes lourdes s'élève à 2,33 (IC à $95 \%[1,24 ; 4,35]$ ) mais avec une hétérogénéité significative entre les sites $(Q=20,1$; $p<0,05)$. Pour les conducteurs de motocyclettes légères, les effectifs d'accidents sont trop faibles pour pouvoir conclure.

\footnotetext{
${ }^{9}$ Concernant la pratique de la remontée de files par la bande d'arrêt d'urgence, celle-ci semble a priori particulièrement à risque. Cependant, le peu d'effectifs d'accidents liés à cette pratique recensés sur les 12 sections étudiées et l'absence de cette pratique sur deux des douze sections étudiées, ne nous a pas permis d'estimer un risque global d'accident. Si l'on ne tient pas compte des deux sections sur lesquelles aucune pratique de remontée de file par la bande d'arrêt d'urgence n'a été observée, le risque d'accident des motocyclistes en remontée de files par la bande d'arrêt d'urgence apparaît comme 8,9 fois supérieur (IC à $95 \%$, [1,8; 45,4]) au risque des motocyclistes circulant dans les voies. Ce résultat est cependant fragile. D'autres investigations seraient nécessaires pour confirmer ce résultat.
}

\section{Interprétations des résultats et discussion}

Les premiers résultats que nous avons présentés concernent la part que représentent les deux-roues motorisés dans le trafic sur les autoroutes urbaines desservant la ville de Marseille. Nous avons vu qu'en heure de pointe, les motocyclettes légères et lourdes représentent $3,4 \%$ du trafic. Cette proportion est nettement plus faible que celle que nous avions observée en 2011 dans le centre-ville de Marseille, qui s'élevait en heure de pointe à $13,8 \%{ }^{10}$ [21].

Concernant les pratiques de remontée de files, nous avons vu que près d'un motocycliste sur trois $(32,1 \%)$ était en train de remonter une file de véhicules lorsqu'il a été observé. Cette proportion est proche de celle observée par Ouellet [9] sur la base de quelques observations faites en 2011 sur trois autoroutes urbaines de Californie. En s'appuyant sur les caméras de vidéosurveillance du réseau autoroutier de Californie, Ouellet montre que, durant les mois de mai, juin

\footnotetext{
${ }^{10}$ Cette différence tient principalement au trafic tous véhicules, qui est bien plus important sur les voies autoroutières que sur les voies urbaines, puisque le nombre de deux-roues motorisés circulant est, lui, similaire (147 véhicules par heure en moyenne dans le centre-ville, 142 sur les voies autoroutières). En revanche, la répartition entre les différentes catégories de deux-roues motorisés diffère nettement, puisqu'en ville nous avions observé que les motocyclettes légères représentaient $60 \%$ des motocyclettes en circulation et les motocyclettes lourdes $40 \%$. Sur les autoroutes urbaines, ces proportions sont respectivement de $24 \%$ et $76 \%$. Les usagers de motocyclettes légères semblent donc nettement moins utiliser les voies autoroutières que ne le font les usagers de motocyclettes lourdes. A contrario, le centre-ville est nettement plus fréquenté par les utilisateurs de motocyclettes légères.
} 
Tableau 4 Effectifs de conducteurs accidentés en remontée de files et hors remontée de files, estimation des kilomètres parcourus par les conducteurs de deux-roues motorisés en remontée de files et hors remontée de files, estimation des risques relatifs et IC à $95 \%$ pour chaque section étudiée sur la période 2004-2011

\begin{tabular}{|c|c|c|c|c|c|c|c|}
\hline $\begin{array}{l}\text { Sections } \\
\text { congestion- } \\
\text { nées }\end{array}$ & $\begin{array}{l}\text { Effectifs de } \\
\text { conducteurs } \\
\text { accidentés en } \\
\text { remontée de } \\
\text { files }\end{array}$ & $\begin{array}{l}\text { Effectifs de } \\
\text { conducteurs } \\
\text { accidentés } \\
\text { hors } \\
\text { remontée de } \\
\text { files }\end{array}$ & $\begin{array}{l}\mathrm{DRM}^{*} \mathrm{x} \text { km } \\
\text { parcourus en } \\
\text { remontée de } \\
\text { files }\end{array}$ & $\begin{array}{l}\text { DRM* }^{*} \text { km } \\
\text { parcourus } \\
\text { hors } \\
\text { remontée de } \\
\text { files }\end{array}$ & $\mathbf{R R}$ & IC à $95 \%$ & \\
\hline Section 1 & 4 & 0 & 312784 & 912938 & - & - & - \\
\hline Section 2 & 1 & 3 & 692232 & 2346393 & 1,13 & 0,12 & 10,86 \\
\hline Section 3 & 3 & 0 & 199780 & 1916079 & - & - & - \\
\hline Section 4 & 1 & 2 & 1643866 & 5135539 & 1,56 & 0,14 & 17,22 \\
\hline Section 5 & 2 & 4 & 655044 & 5004010 & 3,82 & 0,70 & 20,85 \\
\hline Section 6 & 3 & 0 & 1554701 & 3307070 & - & - & - \\
\hline Section 7 & 14 & 10 & 3089125 & 6569849 & 2,98 & 1,32 & 6,70 \\
\hline Section 8 & 0 & 1 & 1546753 & 7211763 & - & - & - \\
\hline Section 9 & 0 & 0 & 2500152 & 1237344 & - & - & - \\
\hline Section 10 & 4 & 1 & 494004 & 2511168 & 20,33 & 2,27 & 181,9 \\
\hline Section 11 & 2 & 2 & 1391069 & 210966 & 0,15 & 0,02 & 1,08 \\
\hline Section 12 & 2 & 2 & 1378595 & 410538 & 0,30 & 0,04 & 2,11 \\
\hline
\end{tabular}

et juillet et au cours des heures de pointe du soir, 40 des 107 (soit $37 \%$ ) motocyclistes circulant, remontaient une file de véhicules au moment où ils ont été observés.

Cette proportion de $32,1 \%$ que nous observons à Marseille, cache cependant des disparités importantes. En premier lieu, les usagers de motocyclettes lourdes remontent significativement plus fréquemment les files que les usagers de motocyclettes légères (respectivement 39,3\% contre $26,8 \%$ ). Cette différence peut s'expliquer par la puissance et la capacité de vitesse plus importantes des motocyclettes lourdes, qui permettent à leurs utilisateurs de remonter les files, même lorsque la vitesse du trafic est assez importante, ce que peuvent plus difficilement faire les utilisateurs de motocyclettes légères. Ensuite, nous avons vu que la proportion de motocyclistes en remontée de files varie fortement en fonction des conditions de circulation. Cela a déjà été observé par ailleurs et de façon plus précise (voir par exemple : [9, 19, 22]). Gemy et Huillet [22] montrent par exemple que lorsque la vitesse du trafic sur la file la plus à gauche est inférieure à $60 \mathrm{~km} / \mathrm{h}$, plus de $90 \%$ des motocyclistes remontent les files de véhicules. D'après la référence [19], la vitesse du flux de trafic influence non seulement la proportion de motocyclistes en remontée de file, mais également la vitesse à laquelle les motocyclistes remontent les files. Lorsque la vitesse est inférieure à
$30 \mathrm{~km} / \mathrm{h}$, les motocyclistes remontent les files à une vitesse en moyenne de $50-55 \mathrm{~km} / \mathrm{h}$. En revanche, lorsque la vitesse du trafic est supérieure à $30 \mathrm{~km} / \mathrm{h}$, ils remontent les files à une vitesse correspondant à la vitesse du trafic augmentée de 20 à $25 \mathrm{~km} / \mathrm{h}$.

Parmi les résultats que nous avons présentés, nous avons également vu que la proportion de motocyclistes dans la circulation remontant les files $(32,1 \%)$ est significativement différente de celle observée dans les accidents (59\%). Ce résultat est discordant avec les résultats issus du programme d'investigations détaillées sur des cas d'accidents de Hurt el al. [5] rapportés par Ouellet [9]. En effet, parmi les accidents de motocycles s'étant produits en 1976 et 1977 sur les voies autoroutières de la ville de Los Angeles et dans des conditions de trafic dense, $29 \%$ des accidents impliquaient un motocycliste en train de remonter les files de véhicules. En revanche, dans les données d'exposition au risque collectées dans le cadre de ce programme quelques années plus tard sur les mêmes sites, $55 \%$ des motocyclistes qui circulaient dans des conditions de circulation similaires, remontaient les files de véhicules. La différence entre les deux proportions est significative (test de Fisher ; $p=0,033$ ) [9]. Soulignons toutefois que ces investigations reposent sur des effectifs très faibles : quatre accidents de remontée de files parmi 14 cas s'étant produits sur une voie 
autoroutière en condition de trafic dense. Concernant les données d'exposition au risque, elles ont pu être collectées sur seulement trois sites et concernent 38 motocyclistes observés (même référence).

Concernant le risque d'accident des conducteurs de deux-roues motorisés remontant les files de véhicules, nous avons montré que sur l'ensemble des sections étudiées, il est 2,63 fois supérieur (IC à $95 \%[1,003 ; 6,89]$ ) au risque des conducteurs ne remontant pas les files. Ce sur-risque pourrait en premier lieu s'expliquer par le différentiel de vitesse entre les motocyclistes remontant les files et les motocyclistes circulant dans les files. Nous n'avons pas mesuré ce différentiel de vitesse au cours de nos observations. Des travaux antérieurs ont cependant mesuré sur un panel d'autoroutes urbaines, y compris sur la région Marseillaise, l'écart entre la vitesse des motocyclistes remontant les files et le flux de trafic (voir notamment [19, 22]). D'après les résultats des investigations réalisées sur la région marseillaise, le différentiel de vitesse s'établit en moyenne à $15,3 \mathrm{~km} / \mathrm{h}$ pour les motocyclistes remontant les files par l'espace interfiles et à $37,9 \mathrm{~km} / \mathrm{h}$ pour ceux empruntant la bande d'arrêt d'urgence. Il peut même atteindre $70 \mathrm{~km} / \mathrm{h}$ dans certains cas. En plus du surcroît de vitesse, la remontée des files de voitures par les usagers de deux-roues motorisés, amène ces usagers à emprunter des espaces de la chaussée (espace interfiles, bande d'arrêt d'urgence, notamment) où ils sont sans doute moins attendus et moins recherchés par les automobilistes que lorsqu'ils circulent dans les files [23]. Ce différentiel de positionnement sur la chaussée par rapport à l'ensemble des autres véhicules, pourrait ainsi contribuer à ce qu'ils soient plus difficilement détectés par les automobilistes, en particulier lors de prises d'informations préalables à des manœuvres de changements de files. C'est d'ailleurs ce que suggère l'analyse des cas d'accidents (voir paragraphe 3.3). Parmi les 36 motocyclistes impliqués dans un accident alors qu'ils remontaient une file de véhicules, 23 entrent en collision avec un autre véhicule qui coupe leur trajectoire et dont le conducteur ne les a pas préalablement détectés. Cette situation ne se retrouve que dans 8 cas sur 25 où le motocycliste circule dans les files. Par ailleurs, remonter les files de véhicules expose les motocyclistes, à davantage d'interactions de ce type avec des usagers susceptibles de ne pas les détecter, par rapport à une circulation dans les files.

Concernant le risque d'accident associé aux différents espaces utilisés par les motocyclistes pour pratiquer la remontée de files, nous avons montré que l'usage de l'espace interfiles est associé à un sur-risque d'accident significatif $(\mathrm{RR}=2,71$ (IC à $95 \%[1,06 ; 6,95])$ ). Comme pour l'ensemble des motocyclistes remontant les files, quel que soit l'espace utilisé, cela pourrait s'expliquer par le différentiel de vitesse entre les motocyclistes en interfiles et les motocyclistes circulant dans les files et par le caractère inattendu pour les automobilistes du positionnement des motocyclistes en interfiles. Mais l'étroitesse de l'espace interfiles peut également contribuer à ce que les motocyclistes confrontés à un véhicule changeant de file soient moins en mesure de l'éviter, par rapport aux motocyclistes circulant dans les files confrontés à un véhicule changeant de file. Les automobiles sont en effet plus proches et l'espace disponible pour effectuer une manœuvre d'évitement, plus réduit. D'autre part, circuler dans l'espace interfiles expose les motocyclistes aux manœuvres de changement de files des véhicules situés à la fois à leur droite et à leur gauche, contrairement aux motocyclistes circulant sur la file la plus à gauche ou sur la file la plus à droite. En ce qui concerne les autres espaces empruntés (bande d'arrêt d'urgence, bande dérasée, etc.) pour remonter les files, nous avons vu que les effectifs d'accidents n'étaient pas suffisants pour pouvoir conclure sur le risque d'accident associé.

\section{Conclusion et perspectives}

Ce travail comporte un certain nombre de limites. En premier lieu, le nombre de sections étudiées est assez faible. Ensuite, les faibles effectifs d'accidents recensés chaque année sur ces sections, nous ont amenés à faire porter les investigations sur plusieurs années (de 2004 à 2011) pour pouvoir disposer d'effectifs suffisants, alors que nous avons pu collecter des données sur le trafic deux-roues motorisés qu'au cours d'une seule année (en 2013). Cette démarche nous a donc conduit à considérer que les conditions de circulation, la part des deux-roues motorisés dans le trafic et les pratiques de remontées de files, notamment, n'ont pas évolué de façon significative entre l'année où les données sur le trafic deux-roues motorisés ont été collectées (2013) et les années où les accidents ont été recensés (2004 à 2011). Ce qui comporte une part d'incertitude.

Malgré le nombre relativement important d'années prises en compte, les effectifs d'accidents recensés ne nous ont pas permis d'estimer des niveaux de risque pour toutes les catégories de motocyclettes, par type de section (sections proches du centre-ville et fortement congestionnées versus sections plus périphériques et moins congestionnées) ou encore en fonction de la gravité des accidents. De ce fait, les risques que nous avons estimés correspondent aux risques d'accident corporel, toutes gravités confondues, des motocyclistes circulant dans des conditions de trafic denses, telles qu'on les rencontre en heure de pointe sur les autoroutes urbaines de l'aire métropolitaine marseillaise. D'autres investigations portant sur un plus grand nombre de sections, d'autres villes notamment, seraient utiles pour confirmer ces résultats. Enfin, il n'est pas à exclure que l'association que nous trouvons entre le fait de remonter les files de voitures et le sur-risque d'accident corporel, soit due à un certain nombre de facteurs de confusion que 
nous n'avons pas contrôlés, comme, par exemple, l'âge des motocyclistes, leur expérience.

Globalement, les travaux présentés dans cet article tendent à montrer que, dans la situation actuelle, et toutes choses égales par ailleurs, les motocyclistes remontant les files de véhicules en heure de pointe sur les autoroutes urbaines de Marseille, ont un risque d'accident significativement supérieur au risque des motocyclistes ne remontant pas les files. Par conséquent, tout accroissement du nombre de kilomètres parcourus par les deux-roues motorisés en remontée de files, aurait probablement pour effet d'augmenter le nombre d'accidents de cette catégorie d'usagers.

\section{Remerciements}

Les auteurs tiennent à remercier M. Boyer du PC CRS-AP de Septèmes-les-Vallons et aux personnes de son service pour leur avoir permis d'accéder aux archives des procès-verbaux d'accidents du secteur étudié. Leurs remerciements s'adressent également aux personnels de la Direction Interdépartementale des Routes Méditerranée pour leur contribution dans la collecte des données de trafic.

\section{Références}

1. Lesteven G (2012) Les stratégies d'adaptation à la congestion automobile dans les grandes métropoles. Analyse à partir des cas de Paris, São Paulo et Mumbai. Thèse de doctorat. Université Paris 1 Panthéon Sorbonne, $370 \mathrm{p}$.

2. Ademe (Agence de l'environnement et de la maîtrise de l'énergie) (2007) Synthèse de l'étude Ademe « deux-roues motorisés Euro 3 : progrès environnementaux et comparaison à l'automobile ». Paris, Ademe, $6 \mathrm{p}$.

3. Aupetit S, Espié S, Pianelli C, et al. (2011) Étude des comportements spontanés de conduites des usagers de deux-roues motorisés dans le trafic urbain et péri-urbain. Rapport de convention DSCR/Ifsttar $n^{\circ}$ 7544. Ministère de l'Écologie, de l'Énergie, du Développement Durable et de la Mer. Paris, 61 p.

4. Hidalgo M, Ragot-Court I, Eyssartier C et al. (2014) Les automobilistes et la circulation inter-files des deux-roues motorisés, attitudes et difficultés pratiques. In : Van Elslande P, Clabaux N (eds.) Actes des journées scientifiques « deux-roues motorisés », 15-16 octobre 2013. Ifsttar, Lyon-Bron, pp. 123-137.

5. Hurt HH, Ouellet JV, Thom DR (1981) Motorcycle accident cause factors and identification of countermeasures. Report DOT-HS-501160, Vol. 1 et Vol. 2. NHTSA. Washington D.C, 425 p. (vol. 1), 404 p. (vol. 2).

6. Clarke DD, Ward PJ, Bartle C et al. (2004) In-depth study of motorcycle accidents. Road safety research report $n^{\circ} 54$, University of Nottingham, Department for Transport. London, $67 \mathrm{p}$.
7. Clabaux N, Fournier JY, Michel JE (2014) Powered two-wheeler drivers' risk of hitting a pedestrian in towns. Journal of Safety Reasearch 51: 1-5.

8. Van Elslande P, Fournier JY, Parraud C (2014) The Safety problems met by powered two-wheelers in urban traffic. Proceedings of the $20^{\text {th }}$ International Conference on Urban Transport and the Environement. Algarave, Portugal, 28-30 may 2014, pp. 479-489.

9. Ouellet JV (2012) Lane splitting on California freeways. Proceedings of the Transportation Research Board 91st Annual Meeting. Paper $\mathrm{n}^{\circ}$ 12-1469. 22-26 january 2012, Washington, DC, $13 \mathrm{p}$.

10. Clabaux N, Michel J-E (2012) Contribution écrite au groupe de travail «Circulation interfiles/équipements de protection individuelle »de l'observatoire interministériel de sécurité routière. Annexe 3.2. Paris, mai 2012.

11. Certu (Centres d'études sur les réseaux, les transports, l'urbanisme et les constructions publiques) (2010) Comprendre le trafic routier. Méthodes et calculs. Centres d'études sur les réseaux, les transports, l'urbanisme et les constructions publiques. Lyon, $111 \mathrm{p}$.

12. Lenguerrand E (2008) L'exposition au risque routier et sa prise en compte dans les analyses épidémiologiques des accidents de la route selon la disponibilité de l'information. Thèse de doctorat. Université Claude-Bernard - Lyon 1, $196 \mathrm{p}$.

13. Joly MF, Joly P, Bergeron J et al. (1991) L'exposition au risque d'accident de la route, un paramètre épidémiologique fondamental et difficile à mesurer. Revue d'Épidémiologie et de Santé Publique $39: 307-313$.

14. Guyot R (2012) Circulation inter-files des deux-roues motorisés. Rapport au ministère de l'intérieur. Ministère de l'intérieur. Paris, $149 \mathrm{p}$.

15. Fleiss JL (1971) Measuring nominal scale agreement among many raters. Psychological Bulletin 76(5) : 378-382.

16. Elvik R, Vaa T (2004) The handbook of road safety measures. Elsevier Science. Amsterdam. 1078 p.

17. Hasselbald V, McCrory DC (1995) Meta-analytic tools for medical decision-making: a practical guide. Medical decision-making 15(1): 81-96.

18. Brenac T (2011) Méta-analyse sur des rapports de taux (taux relatifs). Note non publiée.

19. Certu (Centres d'études sur les réseaux, les transports, l'urbanisme et les constructions publiques) (2012) Observations des remontées de files des 2RM sur VRU de type $A$ en section courante. Centres d'études sur les réseaux, les transports, l'urbanisme et les constructions publiques. Lyon, $27 \mathrm{p}$.

20. Brenac T (1997) L'analyse séquentielle de l'accident de la route (méthode Inrets). Comment la mettre en pratique dans les diagnostics de sécurité routière. Rapport Inrets Outils et Méthodes $n^{\circ} 3$. Institut national de recherche sur les transports et leur sécurité. Arcueil, 79 p.

21. Michel JE, Fournier JY, Clabaux N (2013) Place des deux-roues motorisés dans la circulation urbaine : une étude observationnelle. Transport Environnement Circulation 217 : 58-61.

22. Gemy F, Huillet J (2012) Remontée de files des deux-roues motorisés. Observations sur la zone d'action du CETE Méditerranée. CETE Méditerranée. Aix-en-Provence, $25 \mathrm{p}$.

23. Rogé J, Ferretti J, Devreux G (2010) Conspicuité sensorielle des deux-roues motorisés pendant les manœuvres de remontée de file en fonction de l'âge de l'automobiliste. Le Travail Humain 73 : $7-30$. 